\title{
Discussion to the paper by J. E. Lennard-Jones
}

\section{Chairman: Professor J. C. Goligher}

HADley. We may suspect Crohn's disease on clinical and radiological grounds very strongly indeed-are we ever justified in diagnosing it firmly unless we have got material to look at under a microscope? One can be caught out, Crohn's disease can be mimicked by other things, notably intestinal tuberculosis. This implies laparotomy and biopsy, with its hazards, and if it comes to that how do you stop the surgeon taking it out and destroying the natural history?

LENNARD-JONES. I think this is a very difficult practical problem because to date the diagnosis has only been made on pathological material. Sometimes of course we can make the diagnosis by biopsy particularly if there is a rectal or anal lesion; and I would remind you that in some of these patients biopsy of either of these sites will give you a pathological diagnosis. I think that as clinicians we have got to be prepared now, knowing what we do about the radiological signs and the clinical presentation of this condition, to make a clinical diagnosis. But some times of course we will have genuine doubt and then laparotomy is required to make a diagnosis anatomically. I'd very much like other people's comments on this; I think it is a very important question.

Comment. Could I make just one other point in relation to what Dr Hadley has just said in terms of tuberculosis? Dr Fletcher and I have done a controlled trial Mantoux reaction of Crohn's disease and found that in fact it was contrary to what people previously thought-in fact there is no difference between these and the normal population. Nevertheless I think the Mantoux has an important role in that if negative one can I think pretty safely exclude tuberculosis, and from this point of view this is quite valuable, since now over $50 \%$ of people in the younger age groups will have a negative Mantoux.

HANCOCK. With the DNFB (dinitroflurobenzine) reaction you sensitize the patient to this drug in oil and do skin tests afterwards. Now, I believe this is innocuous, because the chances of being subsequently exposed to this drug are exceedingly rare, and this would settle the question whether in fact they are anergic. Has this been done? Would it be worthwhile?

LENNARD-JONES. The answer to that is that it has been done, and the second answer is that we are just repeating it. We are actually using dinitrochlorobenzine, which obviously is the same thing. There is some data from Bristol, a series I think of about forty patients, suggesting that patients with Crohn's diseases do not become sensitive to this drug. We think this is very interesting and we are just repeating this ourselves, but of course it may be a non-specific thing. We don't know what it is like in colitis, we don't know what it is like in any other disease that I know of. So I think it is a very interesting field but $I$ think at the moment we just can't use it practically.

HANCOCK. Is it a safe test?
LENNARD-JoNes. I think it is a safe test. We've been into this. Of course these people are then sensitive to this drug they've had. The principle of this test is as follows: you have this chemical and you put a spot of it on the skin. Then when, a fortnight or more later, you put another spot on a different area of skin, the patient is now sensitive to it and gets an area of erythema. So that they have a general sensitivity to this compound. Safety really depends on the fact that they won't meet it again, so I think there is a problem here. As far as we can see it is all right; it seems to be of such great practical and theoretical interest that we feel we must test it, but, you know, I feel we have got to keep an open mind about it.

QUESTION. I wonder if we could just have a quick word on the management of anal lesions?

LENNARD-JoNES. Could we ask our Chairman to deal with that?

Chairman. If you care to say anything about the colonic lesions it would be interesting to have a few words on that, I think all of us would like that.

LENNARD-JONES. We've not studied this specifically, and what I am going to say can only be generalities. First of all, sometimes these lesions can be treated in the usual appropriate surgical way and they heal. Sometimes they are not only exceedingly chronic but they are progressive; and we have seen the most disastrous anal lesion spreading right out over the perineum, forward to the groins, and in one recent patient who came to us the whole of the perianal tissues were destroyed and the anus was just sitting there in the middle of this enormous deep ulcerated lesion. In this type of lesion where they don't heal, first of all if it is a minimal lesion (they are often not painful, that is one of their characteristics) one may be able to accept this situation and treat them symptomatically. But if they are causing pain or they are progressive, then obviously more energetic surgical treatment is required. We looked into the matter of doing by-pass operations, say a colostomy or an ileostomy where this is relevant, and in our experience this was unhelpful and lesions tended to persist or progress.

Now, in Lancet $(1967$, ii, 632) there is an exceedingly interesting paper from Stanford University, in which they suggest that by-passing colonic Crohn's disease leads to healing; and our findings and theirs differ in this. The experience at St Mark's generally is that the lesion has to be resected before this type of lesion will heal, and even then it may take an awfully long time.

From the point of view of the physician, sometimes when these lesions have been treated surgically and they take a long time to heal we have found that treatment of the gut disease-sometimes with things like antibacterial agents or steroids, and sometimes local steroids -seemed to accelerate healing. But all these things are rather general impressions; I think they are a difficult problem. 